\title{
Síndrome de Wildervanck (Síndrome cérvico-óculo-acústica)
}

\author{
Wildervanck syndrome
}

João Luís Curvacho Capella', Iris de Souza Yamane², Yoshifumi Yamane ${ }^{3}$

\section{Resumo}

O artigo relata o caso clínico de uma criança do sexo feminino, cinco anos de idade que apresenta a rara síndrome de Wilderwanck que consiste da síndrome de Klippel-Feil associada à síndrome de Duane e deficiência auditiva. O exame revelou alterações marcadas da coluna cervical, limitação bilateral da abdução, limitação da adução do olho direito associada à enoftalmia, diminuição da fenda palpebral, anisotropia em A, esotropia nas lateroversões e hipoacusia auditiva neurosensorial bilateral. Nossa paciente revelou a associação de síndrome de Klippel-Feil do tipo II com síndrome de Duane bilateral.

Descritores: Estrabismo/ diagnóstico; Anormalidades múltiplas/diagnóstico; Síndrome da retração ocular/diagnóstico; Síndrome de Klippel-Feil/diagnóstico; Relatos de casos [Tipo de publicação]

\footnotetext{
'Pós-graduando em Ofalmologia do Serviço de Oftalmologia do Hospital Municipal da Piedade - Rio de Janeiro (RJ), Brasil; ${ }^{2}$ Pós-graduanda do Setor de Cirurgia Refrativa do Serviço de Oftalmologia da Universidade de São Paulo - USP - São Paulo (SP), Brasil; ${ }^{3}$ Doutor; Professor Regente da Disciplina de Oftalmologia da Universidade Gama Filho - Rio de Janeiro (RJ), Brasil.

Recebido para publicação em: 8/4/2008 - Aceito para publicação em 21/7/2008
} 


\section{INTRODUÇÃO}

$\mathbf{E}$ m 1952, Wildervanck ${ }^{(1)}$ descreveu pela primeira vez uma síndrome com acometimento cervical, ocular e auditivo, que consistia na Síndrome de Klippel-Feil ${ }^{(2)}$ associada à Síndrome de Duane $^{(3)}$ e a deficiência auditiva. Estudos subseqüentes mostraram que a Síndrome de Wildervanck afeta preferencialmente mulheres, e pode estar associada à paralisia de outros nervos cranianos inferiores ${ }^{(4-5)}$. Em face da raridade da síndrome e do pequeno número de artigos publicados sobre o assunto, nos estimulou a descrever o primeiro caso da Síndrome de Wildervanck da literatura nacional.

\section{Relato do caso}

Identificação: M.C.C., 5 anos, sexo feminino, branca, natural RJ, estudante.

Queixa principal: "Olho torto para dentro e às vezes para cima”.

História da doença atual: os pais observaram esotropia e hipertropia intermitente no olho direito com 1 ano de idade.

História patológica Pregressa: prematura de $8 \mathrm{~m}$, $50 \mathrm{~cm} / 2,60 \mathrm{~kg}$; membrana hialina; atraso motor e desenvolvimento; surdez bilateral; convulsão afebril; uso de Gardenal.

Ectoscopia: assimetria facial; linha de implantação dos cabelos posterior baixa; pescoço curto com redução do movimento; cifoescoliose; Ombro de Sprengel; torcicolo leve (Figura 1).

Exame externo: epicanto e telecanto.

Biomicroscopia: normal. os olhos.

Acuidade visual sem correção: 0,8 / J1 em ambos

Motilidade (Figura 2): O estudo das excursões oculares revelou limitação da abdução do OD e OE de -3 e limitação da adução do OD de -1 . Observou-se
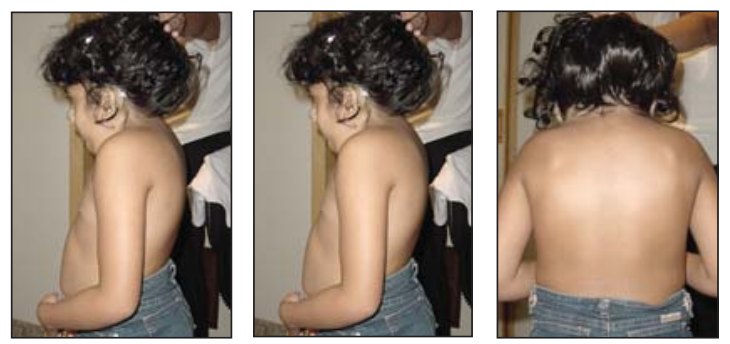

Figura 1: Assimetria facial; linha de implantação dos cabelos posterior baixa; pescoço curto com redução do movimento; cifoescoliose; ombro de Sprengel; torcicolo leve

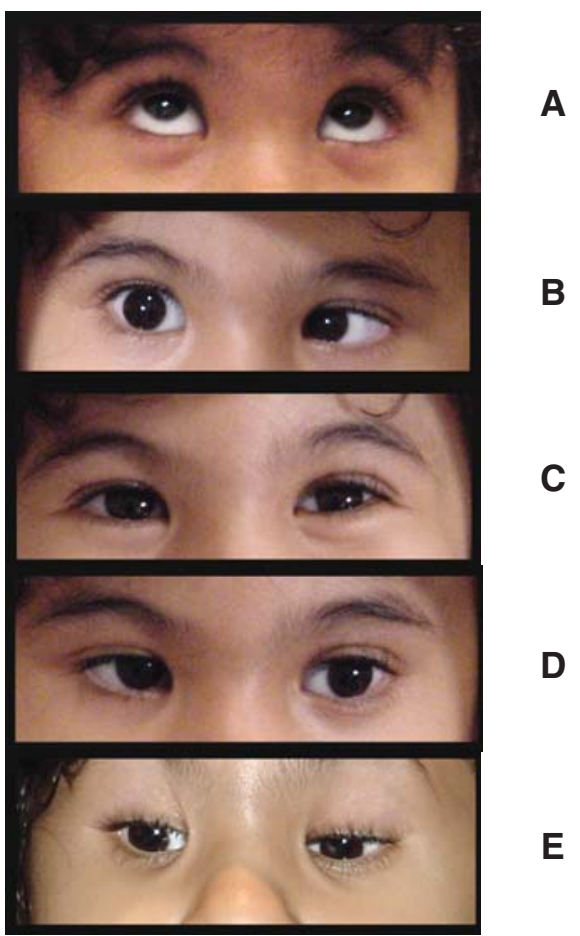

Figura 2: S. Duane com ET em V; B-C: limitação da abdução do OD e OE. C: enoftalmia com diminuição da rima palpebral no OD e $\mathrm{OE}$ na tentativa de adução. Anisotropia em A (A-E)

a diminuição da fenda palpebral e enoftalmia, que acentuava na tentativa de adução do olho direito e anisotropia em "A". O teste de cobertura a $33 \mathrm{~cm}$ revelou as seguintes medidas:

Medida do desvio - $33 \mathrm{~cm}$

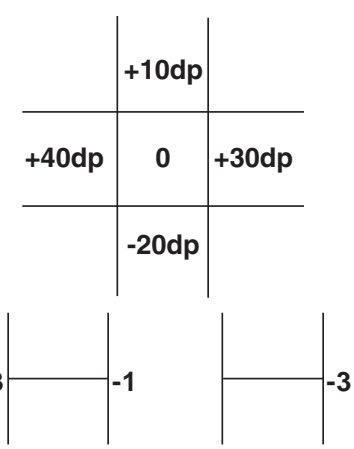

Pupilas: Normais

Fundo de olho: Normal

Tonometria bidigital: Normal

Refratometria:

$+0,25=-1,50 \times 170^{\circ}-\mathrm{AV} 1,0 / \mathrm{J} 1$

$+0,25=-0,50 \times 85^{\circ}-\mathrm{AV} 1,0 / \mathrm{J} 1$ 

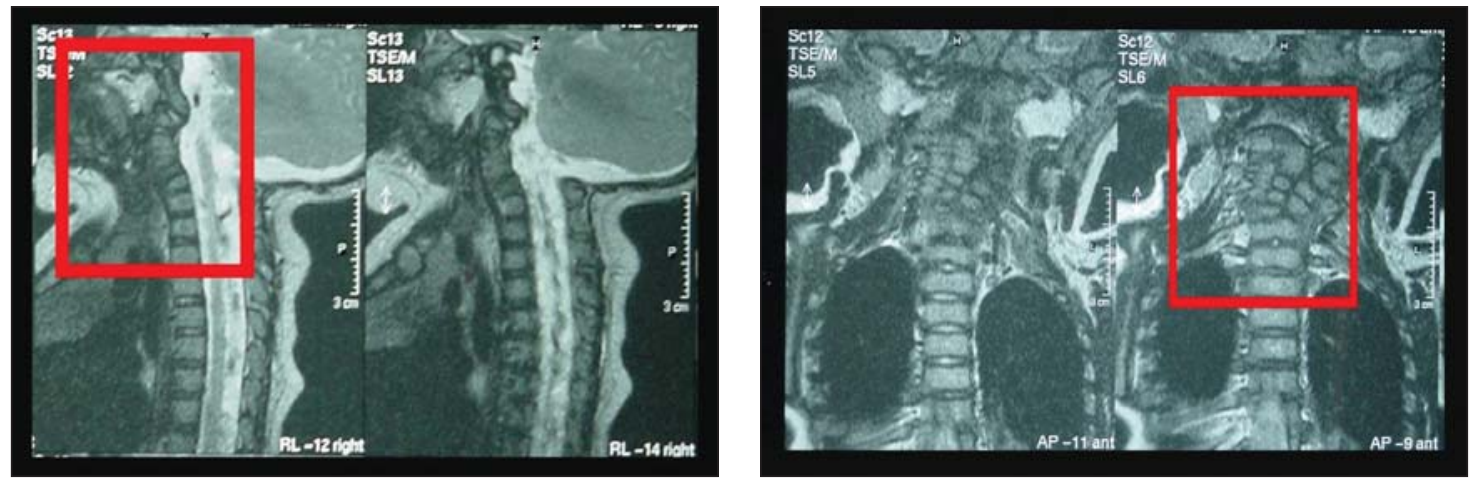

Figura 3: Esquerda: corte sagital: alteração na forma das vértebras cervicais superiores e dos espaços intervertebrais; Direita: corte coronal presença de hemivértebras

Exames complementares realizados:

Radiografias de coluna cervical e torácica com baixa resolução dificultou a identificação de alterações nos corpos vertebrais e nos discos intervertebrais.

Ressonância nuclear magnética de crânio e coluna cervical apresentou alteração na forma dos corpos vertebrais nos cortes sagital e coronal, com fusão do $1^{\circ}$ ou $2^{\circ}$ espaço interespinhal, associada com outras anomalias vertebrais, como hemivértebras (Figura 3).

A eletroencefalografia foi realizada em duas fases diferentes: o primeiro, com sono espontâneo, que apresentou inúmeras ondas agudas e pontas difusas ou nas regiões posteriores. O segundo, com sono induzido, onde se encontrou pontas e ondas agudas esparsas ou difusas. O laudo do neurologista relata sinais de irritação cortical.

O teste de potencial evocado auditivo apresentou ausência de respostas bilateralmente na pesquisa de emissões otoacústicas evocadas. Na audiometria infantil relataram-se curvas audiométricas compatíveis com perda auditiva neurossensorial profunda bilateral (Figuras 4 e 5).

Os exames de ultra-sonografia abdominal e de eletrocardiograma foram normais.

\section{Dıscussão}

A Síndrome de Wildervanck, descrita inicialmente em 1952 por Wildervanck LS, compreende a tríade clínica da Síndrome de Klippel-Feil associada a deficiência auditiva e a Síndrome de Duane (limitação da abdução ou da adução, com retração do globo ocular no olho afetado na tentativa da adução) ${ }^{(1)}$.

Klippel e Feil descreveram o caso de um paciente de 46 anos com "fusão" de vértebras cervicais. Este paciente possuía a tríade clássica da Síndrome de

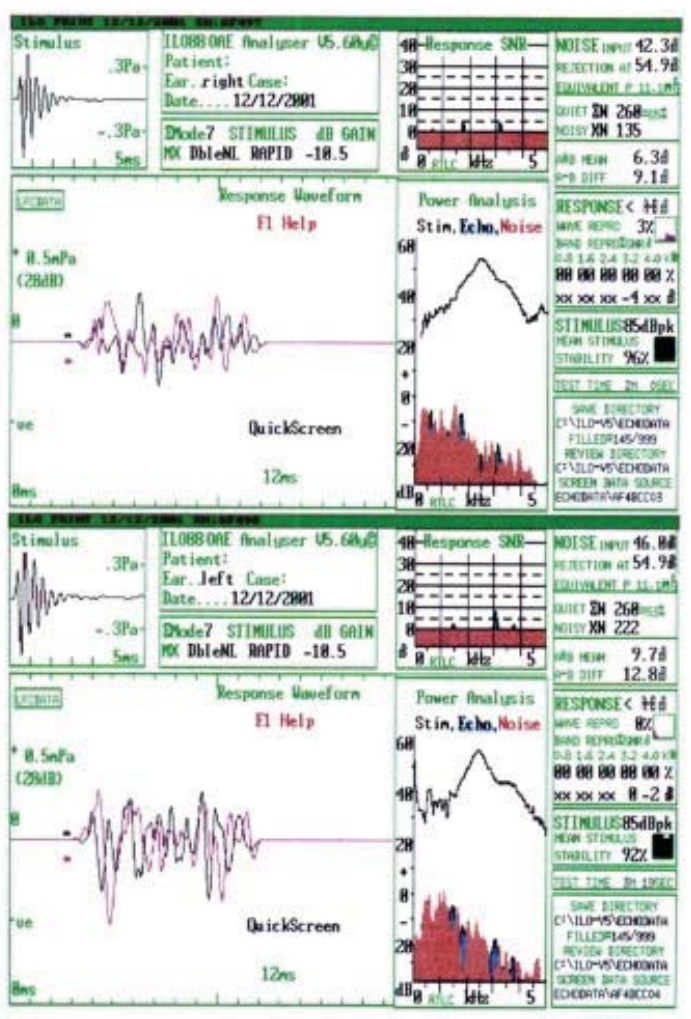

Figura 4. Teste de potencial evocado auditivo revela 3\% de resposta no ouvido direito e $0 \%$ no esquerdo.

Klippel-Feil (KFS): pescoço curto, linha de implantação dos cabelos posterior baixa e redução do movimento do pescoço. $\mathrm{O}$ exame de raios $\mathrm{X}$ da coluna cervical revelava aparente fusão das vértebras cervicais ${ }^{(2)}$.

A incidência da síndrome de Klippel Feil (SKF) é de 1 para 42.000 e pode ser subdividida em 3 tipos 
AUDIOMETRIA TONAL LIMINAR
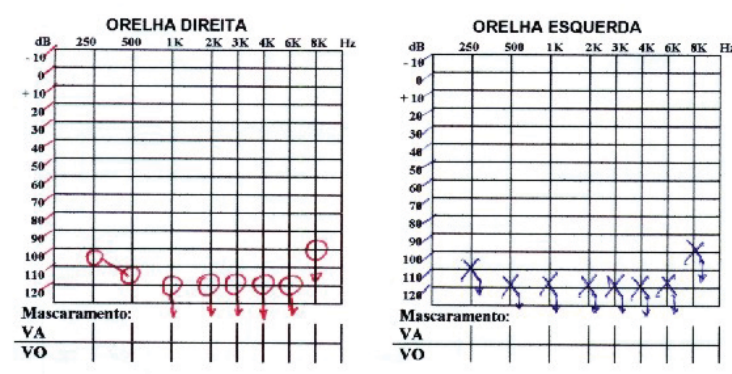

Figura 5: Audiometria infantil - curvas audiométricas mostram perda auditiva neurossensorial profunda bilateral

dependendo da extensão do acometimento das vértebras:

Tipo 1 - Fusão cervical maciça com envolvimento de vértebras torácicas superiores;

Tipo 2 - Fusão do $1^{\circ}$ ou $2^{\circ}$ espaço interespinhal associada com outras anomalias vertebrais, como hemivértebras;

Tipo 3 - Fusão das vértebras cervicais e das vértebras torácicas inferiores ${ }^{(2)}$. tipo II.

Em relação ao nosso paciente, encontramos a SKF

A incidência e a herança desta condição variam com cada tipo de SKF.A incidência de anomalias associadas como o ombro de Sprengel (descolamento congênito da omoplata para cima), fenda palatina, anomalias renais e cardíacas e atraso no desenvolvimento varia com o tipo de KFS. Feil:

Associações freqüentes com síndrome de Klippel-

Escoliose - 60\%; Espinha bífida ${ }^{(6)}-45 \%$; Alterações renais (agenesia renal unilateral vezes ) - 40\%; Ombro de Sprengel - 30\%; Surdez - 30\%; Alterações nas costelas - 30\%; Fenda palatina/ Lábio leporino$15 \%$; Malformações cardíacas - 10\%

Achados menos freqüentes:

Costelas cervicais, anomalias no processo odontóide e na junção atlas-axis, problemas oculares, paralisia do nervo facial, acúmulo de pele no pescoço, assimetria facial, dedos extranumerários ou em falta, obstrução de vias aéreas superiores crônica, má-formação de extremidade superior ${ }^{(2,6)}$.

Achados raros:

MURCS - é a síndrome que se caracteriza por anomalias do ducto de Müller, agenesia renal e displasia do somito cervical. É uma rara associação muito onde a menina afetada revela agenesia úterovaginal associa- da á alterações renais, malformações da coluna cervical e torácica.

VATER - é a síndrome que se associa com anomalia vertebral, atresia anal, fístula traqueoesofagiana e defeitos em raio radial ${ }^{(6)}$.

O sexo feminino responde por $65 \%$ do total dos casos. Os tipos 1 e 3 são os mais freqüentes e se expressam com maior severidade. A incidência para o tipo 2 é igual para ambos os sexos.

A síndrome de Klippel-Feil decorre de fatores genéticos e ambientais . A agressão ocorre entre a terceira e quarta semana do desenvolvimento embrionário, causando imperfeita segmentação dos somitos do mesoderma. Existem relatos esporádicos de casos com herança autossômica dominante ${ }^{(6)}$.

Alterações audiológicas ${ }^{(7)}$ são comuns, porém estudos detalhados destas lesões são poucos elucidativos. McGaughran estudou 44 pacientes portadores de SKF e observou que em $80 \%$ dos casos havia diminuição da acuidade auditiva, unilateral ou bilateral ${ }^{(8)}$. Todos os tipos de alterações auditivas foram encontrados nos pacientes com a síndrome, mas a perda neurossensorial bilateral foi a mais freqüente. Este estudo revelou que em $43 \%$ dos pacientes havia disacusia por perda neurossensorial. Outro achado freqüente foi a hipoacusia auditiva aérea, porém não se observou especificidade nos traçados dos audiogramas para a SKF. Detectou-se também inúmeras anormalidades otológicas como: microtia (estreitamento do meato auditivo externo) microtia) agenesia cóclear (anomalia de Mondini), ausência do vestíbulo anomalias dos ossículos e de canais semicirculares ${ }^{(47-8)}$.

A síndrome de Wildervanck caracteriza-se pela associação da síndrome de Klippel-Feil com deficiência auditiva e à síndrome de Duane. Também pode estar associada a surdez ou surdo-mudez, torcicolo com pescoço curto membranoso, epilepsia, retardamento mental, fenda palatina e destrocardia, esotropia, exotropia, hipertropia, torcicolo ocular, nistagmo horizontal e atrofia corio-retiniana. Afeta principalmente mulheres, mas há relato de homens acometidos ${ }^{(1,4,5,7,9)}$.

A Síndrome de Duane, descrita por Duane caracteriza-se por retração do globo e diminuição da rima palpebral na tentativa de adução devido à co-contração dos retos medial e lateral ${ }^{(3)}$.

Segundo Huber pode-se classificar esta síndrome em:

Tipo I - limitação ou ausência de abdução com adução normal ou levemente limitada e retração do globo ocular com diminuição da rima palpebral na tentativa de adução; 
Tipo II - limitação da adução com abdução normal ou levemente limitada e retração do globo ocular com diminuição da rima palpebral na tentativa de adução;

Tipo III - limitação da adução e da abdução e retração do globo ocular com estreitamento da fenda palpebral na tentativa de adução ${ }^{(10-11)}$. tipos :

Outros sinais podem ser observados entre estes 3

1. Na tentativa de abdução, pode ocorrer um aumento da fenda palpebral;

2. Um desvio vertical anômalo (up-shoot e downshoot) pode ser observado na tentativa de adução do olho afetado.Acredita-se que esse fenômeno vertical seja decorrente de um fenômeno da "rédea", produzido pelo músculo reto lateral espessado que desliza para cima ou para baixo em relação a sua inserção escleral ${ }^{(11)}$.

No nosso caso, encontramos a Síndrome de Duane bilateral.

Indica-se a cirurgia na síndrome de Duane quando o desvio é maior que $20^{r}$ na posiçâo primária do olhar, enoftalmia marcada, torcicolo severo e up shoot ou downshoot intensos ${ }^{(11)}$.

O planejamento cirúrgico ideal é o recuo do(s) Reto(s) horizontal(is) e a cirurgia do fio (faden operation).

No caso descrito, não havia indicação de cirurgia para correção da Síndrome de Duane, pois a enoftalmia, torcicolo e fenômenos verticais anômalos eram discretos.

\section{Abstract}

This article reports a case of a female five years old child with rare syndrome of Wilderwanck wich consists in a association of Klippel-Feil syndrome and Duane syndrome and auditive hypoacusia. Ocular examination reveals alterations of cervical column, bilateral abduction limitations, aduction limitation of right eye associated with enophthalmos, palpebral fissure decrease, anisotropia in A, lateroversions esotropia and bilateral neurosensorial auditive hypoacusia. Our patient reveal an association of Klippel-Feil Syndrome type II with bilateral Duane Syndrome.

Keywords: Strabismus/diagnosis; Abnormalities, multiple/diagnosis; Duane retraction syndromel diagnosis; Klippel-Feil syndrome/diagnosis; Case reports [Publication type]

\section{REFERÊNCIAS}

1. Wildervanck LS. A case of Klippel-Feil's syndrome, associated with abducens paralysis, retraction of the eyeball and deafmutism. Ned Tijdschr Geneeskd. 1952; 96(44): 2752-6.

2. Klippel LM, Feil A. [Anomalie de la colonne vertébrale par absence des vertèbres cervicales; cage thoracique remontant jusqu'à la base du crâne]. Bull Mem Soc Anat Paris. 1912; 87:185-8. French.

3. Duane A. Congenital deficiency of abduction, associated with impairment of abduction, retraction movements, contraction of the palpebral fissure and oblique movements of the eye. Arch Ophthalmol. 1905; 34:133.

4. Brodsky MC, Fray KJ. Brainstem hypoplasia in Wildervanck (cervico-oculo-acoustic) syndrome. Arch Ophthalmol. 1998; 116(3):383-5.

5. Strisciuglio P, Raia V, Di Meo A, Rinaldi E, Andria G Wildervanck's syndrome with bilateral subluxation of lens and facial paralysis. J Med Genet. 1983; 20(1):72-3.

6. Uthoff D. [Klippel-Feil syndrome and status dysraphicus] Klin Monatsbl Augenheilkd. 1986; 189(1):4-6. German.

7. Wang Y, Shi X, Wang Z. [Wildervanck or cervico-oculoacoustic syndrome]. Lin Chuang Er Bi Yan Hou Ke Za Zhi. 1997; 11(11):499-501. Review. Chinese.

8. McGaughran JM, Kuna P, Das V. Audiological abnormalities in the Klippel-Feil syndrome. Arch Dis Child. 1998; 79(4):352-5.

9. Geeraets WJ. Síndromes oculares. 3a ed. São Paulo: Manole; 1979. p. 468.

10. Huber A, Esslen E, Klöti R, Martenet AC. Zum Problem des Duane-Syndroms. Graefe's Arch Clin Exp Ophthalmol. 1964; 167(2):169.

11. Prieto-Diaz J, Souza Dias C. Estrabismo. 2a ed. São Paulo: Roca; 1986. p.339-62.

\section{Errata}

No artigo científico "Síndrome de Wildervanck (Síndrome cérvico-óculo-acústica)" publicado na Revista Brasileira de Oftalmologia na edição de julho-agosto 2008 (Rev Bras Oftalmol. 2008; 67 (4): 188-92), na página 190 , a figura 4 foi publicada com incorreção. 